\title{
El capital social como factor de éxito en las cooperativas mexicanas. Caso: Cooperativa "Las Chiquihuitecas», productora de grana cochinilla
}

\author{
Claudia Leticia Preciado Ortiz \\ Doctorado en Ciencias Administrativas \\ Centro Universitario de Ciencias Económico Administrativas \\ Universidad de Guadalajara \\ José G. Vargas-Hernández \\ Centro Universitario de Ciencias económico administrativas \\ Universidad de Guadalajara
}

Sumario: 1. Introducción. 2. Historia del cooperativismo. 3. Cooperativismo en México. 4. El capital social. 5. Las cooperativas y el capital social. 6. Métodos. 7. La grana-cochinilla: a) Sistema de producción. b) Cría de cochinilla. 8. Análisis de resultados: a) Historia de la cooperativa «Las Chiquihuitecas». b) Formación del grupo. c) Forma de producción. d) Redes. e) Confianza, solidaridad y reciprocidad en el grupo. f) Acción y cooperación. g) Información y comunicación. h) Cohesión social e inclusión. i) Empoderamiento y acción política. 9. Conclusiones. 10. Recomendaciones generales. 11. Referencias bibliográficas.

Resumen: El objetivo principal de este trabajo es reflejar la importancia del capital social en el establecimiento y crecimiento de las cooperativas en México, caso específico la cooperativa "Las Chiquihuitecas», productora de grana-cochinilla (Dactylopius coccus Costa). El método de investigación utilizado fue totalmente cualitativo aplicando el análisis documental y la entrevista a profundidad como instrumento para la recolección de la información. Como resultado se obtuvo que el capital social ha sido el elemento clave en la formación y desarrollo de la cooperativa.

Palabras clave: Capital social, cooperativa, grana-cochinilla.

Abstract: The main objective of this work is to reflect the importance of social capital in the establishment and growth of cooperatives in Mexico, specifically the cooperative "Las Chiquihuitecas», producer of cochineal insect (Dactylopius coccus Costa). The research method used was totally qualitative applying the documentary analysis and the interview in depth as an instrument 
for the collection of the information. As a result, it was obtained that social capital has been the key element in the formation and development of the cooperative.

Keywords: Social capital, cooperative, cochineal insect.

JEL: M000, M100 


\section{Introducción}

En muchas zonas rurales de los países en desarrollo, una gran parte de la población vive por debajo de los niveles de pobreza. La mejora de sus niveles de ingresos y bienestar depende en gran medida de las posibilidades de generar autoempleo productivo y oportunidades de valor agregado de la producción agrícola (Ruben y Heras, 2012; Banco Mundial, 2008; Anriques, 2007).

Las cooperativas establecidas en los países en desarrollo enfrentan frecuentemente problemas, ya que muchas de ellas se establecen sobre la base de criterios políticos por parte de agentes externos, como parte de las estrategias de inversión pública o programas de desarrollo rural lanzados por agencias internacionales y no por los propios agricultores (Ruben y Heras, 2012). Bernard, Gabre-Madhin y Taffese (2007) sostienen que debido a estas políticas gubernamentales la mayoría de las cooperativas presentan entre los miembros un alto nivel de desconfianza, además de las importantes limitaciones a las que se enfrentan para ser más eficaces para mejorar su producción, comercialización y por ende el bienestar de los socios.

Estudios recientes sobre el papel de las cooperativas en la mejora del bienestar de los agricultores alcanzan, sin embargo, resultados contradictorios. Johnson y Berdegué (2007) concluyen que muchas organizaciones cooperativas no están bien integradas en los mercados globales y sólo una pequeña parte de ellas son capaces de alcanzar sus objetivos. Bijman (2007) y Valentino (2003) se refieren tanto a los factores externos (es decir, a la escasa adaptación a las demandas del mercado) como a los factores internos que afectan la organización económica cooperativa y su cohesión social. Bhuyan (2007) afirma con razón que estos factores no son aislados sino interdependientes, lo que implica que las cooperativas deben equilibrar cuidadosamente los intereses de los miembros y los requisitos empresariales.

Otro estudio como el de Francesconi (2008), señaló que las cooperativas etíopes han sido creadas en respuesta a los planes gubernamentales y sólo pretenden atraer subsidios públicos en lugar de ser competitivos en el mercado. Tal cooperación de arriba hacia abajo es probable que induzca una solidaridad real limitada entre los miembros y tiende a disminuir su interés en esfuerzos sustantivos para mejorar la producción y los rendimientos.

Este artículo presenta el análisis de una cooperativa productora de grana cochinilla, enfocando la atención al tema de capital social, las normas sociales que la rigen, la confianza, reciprocidad y las redes internas y externas que tiene. El objetivo central de este trabajo es identi- 
ficar el desarrollo del capital social es una cooperativa y la importancia del mismo en el crecimiento y supervivencia de la asociación.

\section{Historia del cooperativismo}

En 1820 nace en Inglaterra el cooperativismo como un sistema económico, cuando los trabajadores buscan superar sus condiciones sociales, la Cooperativa de Rochdale es mencionada por varios autores como el primer ejercicio de cooperativismo en el mundo, formada por 28 obreros en 1944. Los tejedores se enfrentaban a miserables condiciones de trabajo y bajos salarios, y no podían permitirse los altos precios de los alimentos y los artículos para el hogar. Decidieron que uniendo sus escasos recursos y trabajando juntos podían acceder a los bienes básicos a un precio más bajo.

Inicialmente, sólo había cuatro artículos para la venta: harina, avena, azúcar y mantequilla. Cada cliente se convirtió en miembro y así fue creciendo (International Co-operative Alliance ICA, 2017a). Posteriormente, en 1985 se funda la Alianza Cooperativista Internacional ( $\mathrm{ACl}$, International Co-operative Alliance, ICA, por sus siglas en inglés) estableciendo los principios y valores en las que estaría basado el funcionamiento y desarrollo de las cooperativas (Centro de Estudios Sociales y de Opinión Pública CESOP, 2016). La ICA es una organización no gubernamental independiente que se establece para «unir, representar y servir a las cooperativas de todo el mundo»; sirviendo como vOz y foro mundial para el conocimiento, la experiencia y la acción coordinada para y sobre cooperativas (International Co-operative Alliance ICA, 2017b).

La ICA define cooperativa como «una asociación autónoma de personas unidas voluntariamente para satisfacer sus necesidades y aspiraciones económicas, sociales y culturales comunes a través de una empresa de propiedad conjunta y controlada democráticamente». Los valores que definió como la base de cualquier cooperativa son: autoayuda, auto responsabilidad, democracia, igualdad, equidad, solidaridad, honestidad, apertura, responsabilidad social y el cuidado de los demás. Y los principios son membresía voluntaria y abierta, control democrático de los miembros, participación económica de los miembros, educación, formación e información; cooperación entre las cooperativas y preocupación por la comunidad (ICA, 2017b).

Hoy se estima que el sector tiene alrededor de 1.000 millones de miembros. Las cooperativas emplean, directa o indirectamente, 250 millones de personas en todo el mundo. Las 300 primeras coope- 
rativas del mundo por sí solas tienen una facturación global estimada de 2,53 billones de dólares (2533,1 millones de euros), como lo revela el Monitor Mundial de Cooperativas 2016 (International Co-operative Alliance ICA, 2017b).

\section{Cooperativismo en México}

El cooperativismo en México fue una idea venida de Europa que reflejo los sueños y anhelos de los obreros de la industria mexicana, quienes en la situación de esclavitud que vivían se organizan y forman el Círculo Obreros de México. En 1937 organizan el Primer Taller Cooperativo del que surgiría la primera Sociedad Cooperativa de México (26 sastres de la Cd. de México) (Izquierdo, 2009). En 1939 en Orizaba, Veracruz se fundó la primera Caja de Ahorros con las características de una sociedad cooperativa (Izquierdo, 2009). El ejemplo de la sociedad cooperativa de sastres dio pie a que otras sociedades mutualistas de entonces se convirtieran en cooperativas (carpinteros y sombrereros).

Aunque cabe aclarar que en México en las comunidades indígenas el trabajo colectivo y la propiedad comunal ya existían desde antes, bajo el nombre de calpullis. Bajo revoluciones e inestabilidad política prosperaron las cooperativas en el país y en 1927 apareció en el Diario oficial de la Federación la primera Ley General de Sociedad Cooperativas reconociendo a las cooperativas agrícolas, industriales y de consumo. Acogiéndose el sistema Raiffesen para las cooperativas agrícolas y el sistema Shultse-Delitzch para las industrias, particularmente en el concepto de la responsabilidad de los socios (Izquierdo, 2009).

Tratando de enmendar las deficiencias de la ley anterior, en 1933 en el Diario Oficial se publica un nuevo ordenamiento cuya vigencia fue de 5 años, pues en 1938 se publicó la nueva versión, que estuvo vigente hasta 1994 cuando se da a conocer la Ley General de Sociedades Cooperativas actual (Izquierdo, 2009).

La Ley General de Sociedades Cooperativas publicada en el Diario Oficial de la Federación (DOF) en 1994, es la que regula las empresas cooperativas en México (Centro de Estudios Sociales y de Opinión Pública CESOP, 2016). Según la Ley General de Sociedades Cooperativas (LGSC) de México, artículo 2, menciona que una sociedad cooperativa "es una forma de organización social integrada por personas físicas con base en intereses comunes y en los principios de solidaridad, esfuerzo propio y ayuda mutua, con el propósito de satisfacer necesidades individuales y colectivas, a través de la realización de actividades 
económicas de producción, distribución y consumo de bienes y servicios» (DOF, 1994, pág. 1).

Los principios que deben observar para su funcionamiento son la libertad de asociación y retiro voluntario de los socios, una administración democrática, limitación de intereses a algunas aportaciones de los socios cuando así se pacte, distribución de los rendimiento en proporción a la participación de cada socio, el fomento a la educación cooperativa y en la economía solidaria, participación en la integración cooperativa, respeto al derecho individual de los socios de pertenecer a cualquier partido político o asociación religiosa y la promoción de una cultura ecológica (art. 6, LGSC, DOF, 1994).

Para su constitución, el artículo 11 (LGSC, DOF, 1994) establece que a cada socio se le reconocerá un voto, independientemente de sus aportaciones; será de capital variable, habrá igualdad esencial de derechos y obligaciones de sus socios e igualdad de condiciones para las mujeres, tendrá duración indefinida y se necesitarán mínimo cinco socios para su integración (Fracción V, reformada DOF 13-08-2009, LGSC). Además, la constitución deberá realizarse en asamblea general que celebren los interesados, en la que se levantará un acta con los datos generales de los fundadores y los nombres de las personas que hayan resultado electas para integrar por primera vez consejos y comisiones; así como las bases constitutivas de la misma (DOF, art. 12, LGSC, 1994).

El tipo de régimen (responsabilidad limitada o suplementada) y categoría de la cooperativa (de consumo de bienes y/o servicios, de productores de bienes y/o servicios; y de ahorro y préstamo) también se dejan especificados en el acta (DOF, arts. 14 y 21, LGSC, 1994, respectivamente).

Una vez firmada el acta constitutiva e inscrita en el Registro Público de Comercio que corresponda a su domicilio, las sociedades cooperativas «contarán con personalidad jurídica, tendrán patrimonio propio y podrán celebrar actos y contratos, así como asociarse libremente con otras para la consecución de su objeto social» (DOF, art. 13, LGSC, 1994, pág. 3). Pero también se podrá disolver por «voluntad de dos tercios de los socios, por disminución de los socios a menos de cinco, porque se consumó el objeto de integración, porque la situación económica de la sociedad no le permite seguir operando o por la resolución ejecutoria dictada por los órganos jurisdiccionales que señala el art. 9 de ésta Ley» (DOF, 1994, art. 66, LGSC, pág. 18).

Por la temática de la presente investigación, sólo se define a las sociedades cooperativas de productores, donde entra el caso de estudio. Conforme al artículo 27 de la LGSC (DOF, 1994, págs. 5-6), son socie- 
dades cooperativas de productores, "aquellas cuyos miembros se asocien para trabajar en común en la producción de bienes y/o servicios, aportando su trabajo personal, físico o intelectual. Independientemente del tipo de producción a la que estén dedicadas, estas sociedades podrán almacenar, conservar, transportar y comercializar sus productos, actuando en los términos de esta Ley».

Respecto al funcionamiento y la administración de las sociedades cooperativas, la LGSC establece que su dirección, administración y vigilancia interna, en general, estará a cargo de la asamblea general, el consejo de administración (cuando son sociedades menores a diez socios sólo será necesario nombrar un administrador, art. 43, LGSC, DOF, 1994), el consejo de vigilancia (en sociedades menores a diez integrantes, bastará designar un comisionado de vigilancia, art. 45, LGSC, 1994) y las comisiones y comités que ésta Ley establece y las demás que designe la Asamblea General (art. 34, LGSC, DOF, 1994). De igual forma, las áreas funcionales o divisiones de la cooperativa, se establecerán en función de las actividades que se realizan y lo que sea mejor para el buen desempeño de las mismas (art. 48, LGSC, DOF, 1994).

En cuanto al capital de la cooperativa, éste se conformará primeramente de las aportaciones de los socios por lo menos el valor de un certificado (efectivo, bienes derechos o trabajo) y con los rendimientos que se destinen para incrementarlo conforme a las decisiones de la Asamblea General (DOF, 1994, arts. 49, 50 y 51, LGSC).

\section{El capital social}

Las cooperativas representan una combinación híbrida de una asociación voluntaria y una empresa de negocios (Levi y Davis 2008, Bijman y Hendrikse 2003; Ruben y Heras, 2012). Los miembros de la cooperativa esperan obtener ventajas de pertenecer a un grupo, pero deben comprometerse con las responsabilidades correspondientes. Los compromisos adquiridos se basan en la confianza mutua y reciprocidad entre ellos (Ruben y Heras, 2012).

Las teorías del capital social proporcionan una importante evidencia de que la confianza, la reciprocidad y las redes juegan un papel clave en la superación de las limitaciones de la acción colectiva (Putnam y Col., 1993, Coleman 1987, Grootaert 2001, Ostrom 1994, Woolcock 1998). Además, Olson (1965) argumentó que se requiere cierta coerción e incentivos internos para controlar la conducta libre de conducción. Ostrom $(1994,2000)$ esbozó cuatro elementos esenciales para el análisis de la dinámica del capital social, a saber: (1) confianza y (2) re- 
ciprocidad, (3) redes y (4) reglas o instituciones. La confianza y la reciprocidad constituyen los eslabones fundamentales entre las redes y la acción colectiva y se consideran los factores más relevantes para fomentar la cooperación voluntaria.

El término capital social es un concepto amplio y complejo que ha ido ganando terreno en la investigación social y económica. Según Putnam (2000) su origen data de 1916 con Lyda Judson Hanifan, en un estudio sobre educación rural y el papel de una comunidad involucrada en el éxito de las escuelas, donde anticipó todos los elementos del término, pero su invención no llamó la atención y desapareció sin rastro (Azuero, 2009).

En ese entonces Hanifan definió el capital social como

«aquellos componentes intangibles que cuentan muchísimo en las vidas cotidianas de la gente, es decir la buena voluntad, la camaradería, la empatía y las relaciones sociales entre individuos y familias que conforman una unidad social (...) Si [un individuo establece] contacto con sus vecinos y estos con otros vecinos, se producirá una acumulación de capital social que, posiblemente, satisfaga al instante sus necesidades sociales y entrañe a su vez, un poder social suficiente como para generar una mejora sustantiva en las condiciones de vida de toda la comunidad». (Hanifan, 1916, pág. 130; citado por Lanzini, 2006)

En los ochenta, el psicólogo Pierre Bourdieu presentó el tema de capital social, siendo objeto de un análisis más detallado. A partir de este momento se desarrollaron diversas definiciones y la realización de investigaciones tratando de medirlo. Es cuando Pierre Bourdieu, James Coleman y Robert Putnam se vuelven los precursores clásicos en capital social (Azuero, 2009). Bourdieu (1985, pág. 248) define capital social como «el agregado de los recursos reales o potenciales ligados a la posesión de una red durable de relaciones más o menos institucionalizadas de reconocimiento mutuo».

Posteriormente, el sociólogo James Coleman (1988) lo define como

«los recursos socio estructurales que constituyen un activo de capital para el individuo y facilitan ciertas acciones comunes de quienes conforman esa estructura...El capital social es definido por su función. No es una entidad única sino una variedad de diferentes entidades, con dos elementos en común: todas ellas consisten de algún aspecto de las estructuras sociales, y facilitan la realización de ciertas acciones para los actores, sean personas o actores corporativos, dentro de la estructura». (Coleman, 1990, pág. 103; citado en Azuero, 2009) 
Putnam (1993) conceptualiza éste término en función la pertenencia a asociaciones civiles y los

«aspectos de las organizaciones sociales, tales como las redes, las normas y la confianza, que facilitan la acción y la cooperación para beneficio mutuo. El capital social acrecienta los beneficios de la inversión en capital físico y humano». (Azuero, 2009, pág. 153)

En este momento, Putnam enfatiza el capital social como algo comunitario y no solamente como un elemento individual (Azuero, 2009). Cada uno de estos autores desarrolla sus postulados sobre capital social, pero poniendo su atención en diferentes aspectos teniendo sus trabajos profundas diferencias entre sí, salvo pocas coincidencias muy generales.

El capital social de un grupo social podría que entenderse como «la capacidad efectiva de movilizar productivamente y en beneficio del conjunto, los recursos asociativos que radican en las distintas redes sociales a las que tienen acceso los miembros del grupo en cuestión» (Perlbach, Calderón y Ríos, s.f.). En consecuencia, se puede asumir que el capital social constituye un recurso, Kaztman (2001) menciona que el capital social se refiere, "a los recursos instalados en una red que pueden ser movilizados por quienes participan en ella para el logro de metas individuales y colectivas» (Citado en Atria, 2003, pág. 50). Sunkel (2001; Atria, 2003, pág. 50) establece cinco variables del capital social: participación en redes, reciprocidad, confianza, normas sociales y proactividad.

En cuanto a las aplicaciones de capital social, Rodríguez y Román del Río (2005) mencionan que el capital social se ha utilizado y vinculado en diferentes campos de investigación, tales como desarrollo económico, vida comunitaria, organizaciones y trabajo, democracia, calidad del gobierno, acción colectiva, empresa, vida urbana, familia y comportamiento de la sociedad (jóvenes, educación, escolaridad, salud, medio ambiente, delincuencia, violencia, entre otras tantas áreas).

\section{Las cooperativas y el capital social}

Se han realizado diversos estudios empíricos aplicando las teorías del capital social en el desempeño de las cooperativas.

Durston (1999) realizó un estudio en las comunidades campesinas de Chiquimula en Guatemala sobre como la creación y el fortalecimiento del capital social a través de la implementación de políticas 
públicas podían apoyar en la superación de la pobreza y la exclusión política de comunidades rurales en América Latina. Descubre que las comunidades que fueron objeto de su estudio mostraban una cultura individualista, con poca participación en organizaciones comunitarias; pero tenían una serie de normas claras que les permitían realizar sus prácticas solidarias y recíprocas. Las redes eran compuestas por parientes y vecinos con un ancestro en común. En este lugar se implementó el proyecto PROZACHI, del cual concluye que, aunque parecía que no existiera capital social en las comunidades, a través de la capacitación y apoyo externo se rescataron prácticas del pasado y surgieron oportunidades de crecimiento y desarrollo comunitario (Pejkovic, 2009).

Miranda y Monzó (2003) realizaron un estudio comparativo sobre el capital social y políticas públicas en las localidades campesinas chilenas Cerro Blanco y Ajial de Quiles, por un lado, y La Cruz por el otro. En las primeras descubrieron que el parentesco, la identidad cultural, la vecindad y memoria histórica eran los detonantes del capital social. Además, llegan a la conclusión de que

"cada comunidad reacciona en forma particular a los efectos de las interacciones que tienen lugar entre sus múltiples actores y a las influencias externas de sistemas que se superponen o coexisten, fortaleciendo o debilitando la cooperación comunitaria». (Miranda y Monzó, 2003, pág. 40)

\section{Así como las autoras concluyen}

«que para la superación de la pobreza no alcanza con detectar capital o evaluar el capital social de los grupos, como lo postulan los organismos internacionales de financiamiento, además es preciso comprender, y corregir en los casos que fuere necesario, las formas de articulación entre el Estado y la comunidad». (Pejkovic, 2009, pág. 29)

Perlbach et al. (s.f.) realizaron un estudio sobre la relación del capital social como factor de crecimiento en Mendoza. El indicador utilizado fue que a mayor cantidad de cooperativas se encontraran funcionando en el municipio mayor capital social habría. Su conclusión fue la comprobación del supuesto mencionado, donde había mayor número de cooperativas mayor crecimiento tenía. Ruben y Heras (2012) exploraron el desempeño de las cooperativas de café en Etiopía y su relación con el capital social. Utilizaron cuatro dimensiones diferentes (la confianza, reciprocidad, red interna y externa). 
Los resultados que encontraron fueron que el rendimiento productivo y económico es fuertemente mejorado por el capital social intra-comunitario, pero afectado por el capital social extra-comunitario. Concluyen que las cooperativas con más capital social compartido por sus miembros son más capaces de construir una respuesta comunitaria a las restricciones externas críticas, presentan una mayor resistencia frente a las adversidades y son más capaces de recuperar el acceso a los recursos. Pejkovic (2009) identificó que los precursores del capital social en Río Gallegos, Argentina eran las necesidades y demandas similares de los asociados al momento de incorporarse en una cooperativa, que hubiera relaciones de parentesco y su participación en de las cooperativas en los congresos nacionales de cooperativas. También encontraron que la ausencia de un pasado común, tradiciones o identidades culturales en común constituían una dificultad para el desarrollo de capital social.

\section{Métodos}

El presente trabajo tiene un enfoque cualitativo, donde a través de la comprensión del fenómeno se trata de profundizar en la relación entre el cooperativismo objeto de estudio y la importancia del capital social como factor de éxito. Primeramente, se realizó la investigación documental sobre capital social, su relación con las cooperativas y los estudios empíricos al respecto. De igual forma, se investigó lo correspondiente a la granacochinilla, origen, características, producción, etc. Posteriormente se realizó una entrevista a profundidad con la líder de la cooperativa «Las Chiquihuitecas» para obtener la información de campo.

\section{La grana-cochinilla}

El cultivo de la cochinilla en México es en el estado de Oaxaca, donde los indígenas la cultivaban como actividad complementaria a su agricultura de subsistencia y en la época colonial cobra importancia en la entonces creciente industria textil europea (Tovar, 2000).

La grana cochinilla, es un colorante natural que puede ser utilizado en distintos ramos de la industria, no causa daño alguno al hombre y en la actualidad, por la necesidad de utilizar colorantes naturales en la industria, está teniendo un auge muy significativo.

Los insectos del género Dactylopius se desarrollan en algunas especies de nopal (Tovar, 2000) y producen un compuesto llamado ácido carmínico, el cual principalmente es utilizado en la industria alimenticia 
(lácteos como yogurt y helados, derivados de dulces, goma de mascar, frutas, gelatinas, mermeladas, sopas y salsas), de los cosméticos (para lápiz labial, rubores y sombras), como colorante para teñir textiles naturales y en la medicina (Méndez et al., 1994; Aquino, s.f.).

Existen dos tipos de cochinilla, la silvestre y la fina. La primera es un complejo de ocho especies que crecen como una plaga en las huertas de nopal. Se distingue de la fina porque su cuerpo está cubierto de una madeja de "algodoncillo» blanco difícil de separar de su cuerpo, en vez del polvo blanco de la cochinilla fina fácil de separar. Su colorante es de mala calidad y de baja concentración, contrario al de la fina (Méndez et al., 1994; Tovar, 2000; Aquino, s.f.; Muñóz, 2011). Este insecto vive en diferentes especies de nopales de los géneros Opuntia y Nopalea, últimamente han preferido la variedad Castilla porque no tiene espinas y facilita el manejo de la penca (Tovar, 2000).

Según Muñoz (2011), la industria alimenticia a nivel mundial utiliza el $75 \%$ de la producción. A la industria cosmética se canaliza el 15\% de la producción. El 10\% restante de la producción se reparte entre la industria farmacéutica y la industria textil.

La relevancia del uso de la grana a nivel mundial se debe a que el ácido carmínico producido por la cochinilla fina se caracteriza por su inocuidad en salud pública, su alta estabilidad y poder colorante (Tovar, 2000). Dentro de los factores que intervienen en el desarrollo de este insecto se encuentran vientos directos, la insolación, lluvia y el granizo; así como la temperatura, siendo uno de los factores de mayor importancia sobre el establecimiento, sobrevivencia, crecimiento, desarrollo y reproducción de las cochinillas (Herrera, 1983; Aquino, 1992; citados en Tovar, 2000). De igual forma, se debe de cuidar de insectos depredadores y plagas.

\section{a) Sistema de producción}

Existen dos sistemas básicos de producción: el de planta en pie (cielo abierto y tapexco) y los de penca cortada (micro túnel e invernadero comercial). El tamaño de la explotación determina el sistema de producción a utilizar. El uso de cada uno depende en gran medida de las condiciones ambientales del lugar donde se pretenda establecer la unidad productiva.

El tapexco es más eficiente en sitios protegidos (como cañadas, laderas de cerros, microcuencas etc.) donde las corrientes de aire, tempestades y tolvaneras no son muy fuertes. El micro túnel se recomienda para el sistema de penca cortada y en climas desérticos y semidesérticos, en valles y planicies donde los vientos, tormentas y tolvaneras, así 
como la radiación solar son muy fuertes. El invernadero se recomienda en las mismas regiones que el micro túnel, pero para productores comerciales (Aquino, s.f.).

En Perú y Bolivia, por ejemplo, la producción se lleva a cabo mediante planta en pie por las condiciones ambientales características del lugar (Llanderal y Campos, 2001; Aldama, Llanderal, Soto, y Castillo, 2005). En México se produce a través de los dos sistemas (Aquino, s.f.). Sin embargo, aquí la producción de grana cochinilla se dificulta debido a la existencia de especies de cochinillas silvestres, de enemigos naturales, temperaturas extremas, lluvias fuera de temporada, alta luminosidad y vientos fuertes; por lo tanto, se requieren ambientes acondicionados para controlar dichos factores, mediante cobertizos, microtúneles o invernaderos y obtener buenas cosechas (Llanderal y Campos, 2001; Aldama, Llanderal, Soto, y Castillo, 2005).

Para el cultivo de la cochinilla, cuando es con penca cortada, ésta se lleva a un lugar protegido para realizar su infestación de grana y posteriormente colgarse para completar el ciclo de aproximadamente 90 a 120 días y se convierta en adulto. Se recomienda dar muerte rápida a la cochinilla para acelerar el secado y así evitar pérdida en peso y carmín. Algunos métodos son: inmersión en agua recién hervida, aplicación de vapor de agua, por congelación, aspersión con hexano al $100 \%$ de pureza y por asfixia (Tovar, 2000).

Posteriormente, el secado de la cochinilla se realiza utilizando secadores solares, estufas con focos, sol directo o secarse a la sombre, cuidando que el sitio donde se realice está actividad esté completamente seco (Tovar, 2000). Posteriormente se realiza la clasificación, limpieza y empaque del producto. El método más utilizado es el tamizado. Una vez seca, limpia, clasificada se guarda en bolsas de polipropileno, siendo la cantidad por bolsa de 50 kg a 70 kg (Quintanilla, 19996; citado en Tovar, 2000).

\section{b) Cría de cochinilla}

Existen dos formas de iniciar la cría de la cochinilla. La primera es colocar los nidos conteniendo el pie de cría y dejándolos ovopositar hasta que mueran las hembras reproductoras. El segundo caso se refiere a llenar nidos con bastante cochinilla, para infestar las pencas y rotarla al menos 6 pencas por nido, en este caso, se van cambiando a pencas nuevas cada tercer o cuarto día dependiendo de que se logre la adecuada infestación del cladodio, lo cual puede ser cuando vea al menos 100 insectos en un cuadro de $10 \times 10 \mathrm{~cm}$ (Aquino, s.f.). 
Proceso de producción (Muñoz, 2011):

1. Plantación del nopal con los cuidados correspondientes, fumigación, riego y revisión constante.

2. Cortar la tercera penca del nopal, ya que es la que cuenta con más nutrientes y llevarla al invernadero.

3. Poner camas de pencas y colocar la grana cochinilla en ellas para que se instalen en ellas.

4. Una vez que la cochinilla definió su penca, colgar las pencas con ganchos dentro del invernadero para que la grana cochinilla se alimente, crezca y se reproduzca, el tiempo de vida de las hembras es de 3 meses, mientras que los machos solo viven 60 días.

5. Se instala una nueva cama de pencas para que cuando se reproduzcan, las pequeñas ninfas caigan en ellas, se instalen y comience nuevamente el proceso.

6. Cuando pasan los 3 meses de vida de las ninfas, se colectan penca por penca, se limpian y empacan para la venta.

\section{Análisis de resultados}

a) Historia de la cooperativa «Las Chiquihuitecas»

La cooperativa "Las Chiquihuitecas» es una cooperativa registrada bajo el régimen de sociedad de responsabilidad limitada («los socios solamente se obligan al pago de los certificados de aportación que hubieren suscrito» art. 14, LGSC, pág. 3, DOF, 1994) de capital variable ubicada en la localidad de Casa de Piedra perteneciente al Municipio de Autlán de Navarro, Jalisco. Cuyo objetivo es la producción de grana-cochinilla. Es una cooperativa de carácter ordinaria, según los artículos 30 y 31 de la LGSC (DOF, 1994), puesto que para su funcionamiento sólo fue necesario su constitución legal.

\section{b) Formación del grupo}

Inició operaciones en el año 2011 con un grupo de mujeres (10) con el objetivo de generar autoempleo y propiciar el desarrollo económico local. La inquietud nace de una persona ajena a la localidad que las motiva e incentiva para participar en el Fondo Nacional de Apoyo para las Empresas de Solidaridad (FONAES). 
Por problemas de desconfianza e incumplimiento de algunas cuestiones, una vez que el grupo comienza actividades se comienza a desintegrar, quedando actualmente 6 personas.

La estructura que tienen es la establecida por la Ley General de Sociedades Cooperativas, un representante legal, un secretario y tesorero, un miembro del consejo de vigilancia y tres vocales. A raíz de que el grupo se reconfiguró, actualmente están en trámites para dar de baja el acta constitutiva inicial y dar de alta los integrantes actuales que son los que realmente llevan a cabo las actividades. Y cuentan con dos personas externas al grupo, que son de planta que les ayudan en las actividades para funcionamiento del invernadero (acarrear pencas, limpiar el nopal, enganchar las pencas, aseo, entre otras).

El tipo de producción que manejan es por invernadero, que tiene una extensión de 2.500 metros cuadrados. La cantidad producida varía dependiendo del espacio aprovechado, pero está entre $5 \mathrm{~kg}$ y $150 \mathrm{~kg}$ aproximadamente cada 3 meses.

La comercialización del producto desde un principio un ingeniero les compraba directamente la producción y él se encargaba de la venta del producto. Actualmente, están buscando por su cuenta clientes para venderles directamente ellas la producción.

\section{c) Forma de producción}

1. El plantío de nopal lo tienen en un terreno cerca del invernadero, hay que mantenerlo limpio y libre de plaga.

2. De este plantío se seleccionan las mejores pencas de nopal para la infestación de cochinilla. Se cortan y se limpian de espinas y se lavan para que no tengan cochinilla silvestre y entre contaminado al invernadero y propague ésta y haga que se pierda la producción de cochinilla fina.

3. Una vez limpia la penca se introduce en el invernadero, se engancha y se espera a que se haga la infestación y por ende el cultivo de cochinilla fina.

4. Los cuidados que deben de tener en el invernadero es tenerlo a puerta cerrada para que no entren esporas de cochinilla silvestre y contamine la producción. No entrar con ropa negra porque altera a los machos ni con perfume en la piel.

5. Cuando se llega el tiempo de la recolección, se limpia la cochinilla y se tamiza. Posteriormente, se deja secar para ser empaquetada. Mencionaba la líder que se guarda en bolsas negras para que no pierda su color. 
El precio de venta es consultado en internet para ver a como se está cotizando en el mercado, oscila entre 30 dólares a 35 dólares por kilo, generalmente. Pero en periodos de invierno llega hasta a 80 dólares el kilo.

La entrevista se realizó a la Sra. Irma Dolores Mondragón Sandoval, líder del grupo, aunque actualmente no funja como representante legal. Respecto a las decisiones, menciona que las decisiones se toman en conjunto y por votación eligen el curso de acción a seguir.

Al cuestionarla sobre la interacción del grupo con otros dentro y fuera de la comunidad, con objetivos similares, menciono a Campo Carmín, S.P.R. de R.L., sociedad también dedicada a la producción de grana cochinilla en invernadero en Morelos, quienes han sido la fuente más importante de asesoramiento para echar a andar el proyecto. También mencionó a otros grupos con objetivos diferentes pero que las han apoyado mucho para seguir adelante, tales como la Junta Intermunicipal del Rio Ayuquila (JIRA), el Centro Universitario de la Costa Sur (CUCSUR), el H. Ayuntamiento de Autlán de Navarro, Jalisco; y la Comisión Nacional de Áreas Naturales Protegidas (CONANP).

Todo esto respaldado por el art. 60 de la LGSC que dice «Las sociedades cooperativas, podrán recibir de personas físicas y morales, públicas o privadas, nacionales o internacionales, donaciones, subsidios, herencias y legados para aumentar su patrimonio» (DOF, 1994, pág. 16).

En cuanto al financiamiento, su principal fuente es la aportación de los socios, el proyecto inicial que las financió, la venta del producto y la participación en proyectos del gobierno. Sin embargo, es uno de sus principales problemas actualmente.

\section{d) Redes}

Las redes de intercambio social son, pues, una condición necesaria para la reciprocidad y la confianza (Ruben y Heras, 2012). Las redes apoyan la reciprocidad mediante la repetición de acciones confiables y el intercambio de información entre individuos. Pueden ser estructuradas vertical o horizontalmente, basadas en el intercambio entre pares o por conexiones jerárquicas (Gittell y Vidal 1998). En México, la LGSC en su artículo 74, promueve la formación de redes entre las cooperativas para su beneficio, estableciendo que «las Sociedades Cooperativas de producción y de consumo se podrán agrupar libremente en Federaciones, uniones o en cualquier otra figura asociativa con reconocimiento legal» (DOF, 1994, pág. 19). 
Se le cuestionó sobre el número de amigos o familiares cercanos que trabajaban en el grupo, a lo que respondió:

"Todos somos amigos, la representante legal es mi amiga y conocida, dos de los integrantes son mis hijos, mi concuña, otra amiga y una servidora; somos los que actualmente estamos comprometidos con el proyecto...»

Sobre la participación en redes de otras cooperativas, aún no están integradas a ninguna.

\section{e) Confianza, solidaridad y reciprocidad en el grupo}

Paldam y Svendsen (2000) afirman que la alta confianza y la reciprocidad son vitales para controlar la libertad de circulación. Las cooperativas agrícolas mejor organizadas y más integradas son también más fiables frente a las agencias de los mercados financieros $y$, por lo tanto, tienen un acceso más fácil a la financiación (Portes y Sensenbrenner, 1993).

La Sra. Irma menciona que la confianza es el elemento clave que ha hecho prosperar al grupo, así como el compromiso y la ayuda mutua entre ellos. Cuando se realizan acuerdos, éstos son verbales y se cumplen en su totalidad, si alguno no puede llevarlos a cabo avisa con tiempo para no afectar al grupo y se compromete a sustituir a otro miembro en un futuro cuando alguno de los otros no pueda apoyar.

Además, considerando lo que la LGSC establece (art. 64, 1994) que, en las sociedades cooperativas de productores, la prestación del trabajo personal de los socios podrá ser físico, intelectual o de ambos géneros, se organizan de tal manera que, si en algún momento alguien no puede apoyar en trabajos físicos por tiempo o salud, se turnan o reemplazan las actividades por otro miembro del grupo o personal externo asalariado (conforme al art. 65, LGSC, DOF, 1994).

Por otra parte, como aún la cooperativa no tiene una producción fija y por ende un ingreso fijo, menciona que si alguno de los miembros tiene un incentivo económico mayor fuera de la cooperativa lo más obvio es que tienda a abandonar el grupo.

\section{f) Acción colectiva y cooperación}

Se les preguntó si en los últimos doce meses se habían involucrado en alguna actividad en beneficio de la localidad, la respuesta fue positiva en actividades de reforestación y recolección de basura. 
Menciona que no todas las personas de la comunidad se involucran con la total disposición y tienden a ser criticadas por las mismas personas de la comunidad que si participan.

\section{g) Información y comunicación}

Las tres fuentes más importantes de información que se utilizan para tomar decisiones en el grupo es a través de las reuniones y asesoría externa. JIRA es la organización externa que las apoya proporcionándoles información sobre proyectos de financiamiento en los que pueden participar y les apoya asesorándolas en los trámites.

\section{h) Cohesión social e inclusión}

La heterogeneidad puede convertirse en un impedimento importante para la cooperación (Putnam 2000), ya que la posibilidad de conectarse con redes externas reduce la probabilidad de interacción entre miembros dentro de la misma cooperativa.

La sensación de unión o cercanía del grupo con su comunidad en muy fuerte, así como entre ellos donde la relación va más allá de lo laboral. Respecto a la existencia de diferencias entre los miembros del grupo y la comunidad, son mínimas y no causan problemas.

En cuanto a la frecuencia de reuniones del grupo, sólo se reúnen cada que hay que tomar decisiones importantes, de lo contrario la líder decide e informa lo realizado.

\section{i) Empoderamiento y acción política}

Es muy feliz y por lo tanto considera tener un control total e sus decisiones y de cambiar el rumbo de su vida. Por lo tanto, considera tener un gran impacto en mejorar su localidad y el grupo como tal.

En los últimos 12 meses se ha reunido más de 5 veces junto con personas de la localidad para hablar con el gobierno y realizar peticiones en beneficio de la comunidad.

En la cual la mayoría de las peticiones a tenido éxito. 


\section{j) Problemática actual}

1. El principal problema que tienen actualmente es la falta de mano de obra para el corte de pencas. Esto les ocasiona que desde su inicio a la fecha no han podido utilizar el invernadero en su máxima capacidad porque no hay personas que quieran trabajar cortando pencas de nopal debido a lo difícil de la actividad pues terminan ahuatados de todo el cuerpo.

2. El segundo problema es la falta de financiamiento para la operación y mantenimiento del proyecto.

3. Falta de capacitación a los miembros actuales en cuestiones de administración y mercadeo, de manera que ellos sean autosuficientes y busquen su mercado, en lugar de tener intermediarios que se lleven la mayor parte de sus utilidades.

\section{Conclusiones}

Primeramente, respecto al producto se puede concluir que la producción de grana-cochinilla es una oportunidad de negocio porque: es un producto no perecedero (la cochinilla seca) que se puede almacenar hasta obtener los volúmenes adecuados para su venta y sea más rentable. Es de fácil almacenamiento y en épocas de escasez como son los meses de invierno puede venderse a un precio considerablemente superior al del precio promedio anual.

Es una alternativa de autoempleo, pues si selecciona un buen sistema de cría combinado con mano de obra familiar, materiales e instrumentos de la región, y se participan en apoyos gubernamentales, los ingresos serían considerables, al reducir los costos de inversión y producción. Así como se aprovecharían las zonas áridas y semiáridas del país en beneficio de los habitantes de esas zonas. De igual forma, al ser un producto natural, no tóxico y totalmente orgánico, es una excelente alternativa para sustituir los colorantes artificiales, y aquí está el negocio.

Sin embargo, dentro de las desventajas que tiene la producción de este artículo, es que por ser algo relativamente nuevo hay mucho desconocimiento sobre su cultivo y manejo, así como su mercado y venta. Además, la actividad de corte de pencas es complicada y no cualquier persona lo sabe y quiere realizar, por las consecuencias que acarrea de terminar todo ahuatado. Y debe ser producido en grandes volúmenes para que sea rentable todo el sistema productivo e inversión.

Referente al capital social, con la entrevista se pudo constatar la importancia del capital social en la formación de cualquier grupo, y en 
este caso de la cooperativa. Las redes y relaciones que inconscientemente han formado con el CUCSUR, con JIRA y el H. Ayuntamiento, han sido factores muy importantes en que esta empresa siga a flote, por los diversos apoyos que han recibido y siguen recibiendo, tales como capacitación hasta asesorías para participar en proyectos económicos que regulen la situación en tiempos de crisis y poca producción. Tal como lo mencionan Perlbach et al. (s.f.) que el capital social no se forma por un solo individuo sino por la sociabilidad e interacción con otros y las redes de relaciones aparecen como claves entre los individuos y grupos.

La confianza, solidaridad, reciprocidad, acción colectiva y cooperación entre los miembros son otros elementos importantísimos en el desarrollo y funcionamiento de la cooperativa. La manera en cómo se turnan y organizan los trabajos, los horarios, la confianza mutua de saber que cuentas con la persona para sacar el trabajo, el apoyo, la familiaridad y amistad fortalecen los lazos y vínculos dentro del grupo para mejor funcionamiento y cohesión entre ellos.

Perlbach et al. (s.f.) establecen que la reciprocidad es fuerte cuando los integrantes de un grupo se preocupan por los intereses de todos. Y éste elemento se manifiesta no con la aplicación inmediata del contrato legal que los unió, sino el altruismo a corto y mediano plazo y el beneficio personal como resultado de lo anterior.

Otro factor importante es el liderazgo que ejerce la Sra. Irma en la dirección de este grupo, pues a pesar de no ser la representante legal por el momento, todos los miembros se subordinan ante ella en un ambiente de confianza y compromiso por sacar adelante el proyecto. Además, de que todos comparten una misma visión de apoyo a la comunidad y participar en actividades que beneficien a todos. En esta parte se manifiesta la proactividad, un involucramiento activo y comprometido con el grupo y la comunidad. El capital social se forma con personas activas, creativas, movidas y no como entes pasivos receptores de productos, servicios o derechos.

\section{Recomendaciones generales}

Como alternativas de solución a su problema de capacitación, se sugiere que aprovechando la excelente relación que se tiene con las autoridades del Centro Universitario de la Costa Sur (CUCSUR) se solicite el establecimiento de un convenio de prácticas profesionales con las carreras de Ingeniero en Agronomía e Ingeniero en Recursos Naturales para que los jóvenes las apoyen en asesorías correspondientes al 
cuidado de plagas de manera natural, así como la manera de eficientar la producción de grana-cochinilla sin contaminar los procesos.

De igual forma, un convenio de prácticas profesionales con las carreras de Administración, contaduría e informática para que apoyen a las personas del grupo con cursos de capacitación en cuestiones administrativas, contables y manejo de la computadora y búsquedas en internet, de manera que sean autosuficientes y se empoderen del importante negocio que emprenden.

\section{Referencias bibliográficas}

Aldama, A.C., Llanderal, C.C., Soto, H.M. y Castillo, M.L. (2005). Producción de Grana-Cochinilla (Dactylopius coccus Costa) en plantas de nopal a la intemperie y en microtúneles. Ensayo. Agrociencia. 39. 161-171.

AnRIQUES, G. (2007). Rural development and poverty reduction: is agriculture still the key? Agricultural Development Economics, 4(1), 5-46.

Aquino, P.G. (s.f.). Producción de Grana Cochinilla. Secretaría de Agricultura, Ganadería, Desarrollo Rural, Pesca y Alimentación (SAGARPA). Subsecretaría de Desarrollo Rural. Dirección General de Apoyos para el Desarrollo Rural. Sistema de Agronegocios de Traspatio. Recuperado de: http://www. sagarpa.gob.mx/desarrolloRural/Documents/fichasaapt/Producción\%20 de\%20Grana\%20Cochinilla.pdf

ATRIA, R. (2003). La dinámica del desarrollo del capital social: factores principales y su relación con movimientos sociales. En ArRiAgA, I. y MiRANDA, F. (Compiladoras). Capital social: potencialidades analíticas y metodológicas para la superación de la pobreza (págs. 49-62). Seminario taller «Capital social, una herramienta para los programas de superación de la pobreza urbana y rural». Comisión Económica para América Latina. (CEPAL). División de Desarrollo Social. Naciones Unidas. Santiago de Chile, 8 y 9 de enero de 2003.

AzUero, R.A. (Ene-Jun, 2009). Capital social e Inclusión Social: algunos elementos para la política social en Colombia. Cuadernos de Administración. Universidad del Valle. Núm. 41. Recuperado de http://www.scielo.org.co/pdf/ cuadm/n41/n41a11.pdf

Banco Mundial (2008). Development Report 2008: Agriculture for Development, Washington DC: The World Bank.

Bernard T., Gabre-Madhin, E. and Taffese, A.E. (2007). Smallholders commercialization through cooperatives: a diagnostic for Ethiopia, Discussion Paper No. 722. Washington DC: International Food Policy Research Institute (IFPRI).

BHUYAN, S. (2007). The «people» factor in cooperatives: an analysis of members' attitudes and behavior. Canadian Journal of Agricultural Economics, 55, 275-298. 
BIJMAN, J. (2007). How can cooperatives meet the challenges of agrifood supply chains? In Ton, G., BIJMAN, J. and OORTHUIZEN, J., eds, Producer Organizations and Market Chain, Wageningen: Wageningen Academic Publishers, pp. 91-116.

BIJMAN, J. and HeNDRIKSE, G. (2003). Co-operatives in chains: institutional restructuring in the Dutch fruit and vegetables industry, Research Paper ERS-2003089-ORG Rotterdam, Erasmus Research Institute of Management (ERIM).

Bourdieu, P. (1980). Le Capital Social, Notes Provisoires. Actes de la Recherche en Sciences Sociales. No. 31 Janvier, 2-3.

Centro de Estudios Sociales y de Opinión Pública (CESOP) (junio, 2016). Cooperativismo en México. Cámara de Diputados LXIII Legislatura. Documento de trabajo núm. 217. Recuperado de www.diputados.gob.mx/cesop

Coleman, J. (1987). Norms as social capital. in Radnitzky G. and Bernholz P., eds, The Economic Approach Applied Outside the Field of Economics, New York: Paragon House, pp. 133-155.

Coleman, 1. S. (1988). Social Capital in the Creation of Human Capital. The American Journal of Sociology, Vol. 94, Supplement: Organizations and Institutions: Sociological and Economic Approaches to the Analysis of Social Structure, S95-S 120.

Francesconi, G.N. (2008). Cooperation for competition linking Ethiopian farmers to markets, PhD Thesis, Wageningen: Wageningen Academic Publishers.

GITTELL, R. and VIDAL, A. (1998). Community Organizing: Building Social Capital as a Development Strategy, Thousand Oaks CA: Sage.

Grootaert, C. (1998). Social Capital: The Missing Link? Washington DC, The World Bank.

Grootaert, C. (2001). Does social capital help the poor? A synthesis of findings from the local level institutions studies in Bolivia, Burkina Faso, Working Paper No. 10. Local Level Institutions, World Bank, Washington, DC.

Grootaert, C., Narayan, D., Jones, V. and Woolock, M. (2004). Measuring Social Capital: an Integrated Questionnaire, Washington DC: World Bank.

International Co-operative Alliance (ICA) (2017a). History co-operative movement. Recuperado de http://ica.coop/en/whats-co-op/history-co-operativemovement

INTERNATIONAL Co-operative Alliance (ICA). (2017b). What is a cooperative?. Recuperado de http://ica.coop/en/what-co-operative

Izquierdo, M.M. (2009). «Problemas de las empresas cooperativas en México que atentan contra su naturaleza especial». Boletín de la Asociación Internacional de Derecho Cooperativo. Núm. 43, Bilbao, España, págs. 93-123. Recuperado de https://dialnet.unirioja.es/servlet/articulo?codigo=3074487

Johnson, N. and Berdegue, J. (2004). Collective action and property rights in R. Meinzen-Dick and M. DI Gregorio, Collective Action and Property Rights for Sustainable Development, brief 13; 2020 Focus No. 11.

KazTMAN, R. (2001). Segregación Residencial y Desigualdades Sociales. Sistemas de Información, Monitoreo y Evaluación de programas Sociales. SIEMPRO, Buenos Aires. 
LANZINI, E. (2006). Capital social, una herramienta básica para el desarrollo local. In: Seminario Internacional Desarrollo Económico Territorial y Empleo (3. ${ }^{\circ}, 2006$, Montevideo). Agentes y agenda para el desarrollo económico territorial y el empleo; trabajos presentados. Montevideo, Red Desarrollo Territorial y Empleo (DETE). 1 disco compacto, $8 \mathrm{~mm}$. Recuperado de http:// www.congresos-rohr.com/IIISEMDETE2006/docs/tls/02_Lanzini.pdf

DOF (Diario Oficial de la Federación) (1994, 03 de agosto). Ley General de Sociedades Cooperativas (LGSC) (1994). Título I, Capítulo Uno, Disposiciones Generales. Cámara de Diputados del H. Congreso de la Unión. Recuperado de http://www.diputados.gob.mx/LeyesBiblio/pdf/143.pdf

MuÑoz, P.E. (abril 2011). Proyecto de inversión para la empresa Productores y comercializadores de Grana Cochinilla del estado de Querétaro, S.C. DE R.L. Memoria de Estadía Final para obtener el título de Ingeniero en Innovación y Desarrollo Empresarial. Universidad Tecnológica de Querétaro. Santiago, Querétaro.

OLson, M. (1965). The Logic of Collective Action, Cambridge, MA: Harvard University Press.

Ostrom, E. (1994). Constituting social capital and collective action, Journal of Theoretical Politics, 6(4), 527-562.

Ostrom, E. (2000). Collective action and the evolution of social norms, Journal of Economic Perspectives, 14(3), 137-158.

Paldam, M. and SVEndsen, G.T. (2000). Missing social capital and the transition in Eastern Europe, Working Papers 00-5, University of Aarhus, Aarhus School of Business, Department of Economics.

Pejkovic, C. (2009). Políticas sociales, cooperativas y capital social: Estudio sobre la promoción de cooperativas de trabajo en la ciudad de Río Gallegos. Tesis presentada para la obtención del grado de Licenciada en Sociología. Universidad Nacional de la Plata. Recuperado de: http://www.memoria. fahce.unlp.edu.ar/tesis/te.585/te.585.pdf

Perlbach, I., Calderón, M. y Ríos, R.M. (s.f.). Un indicador de capital social como factor del crecimiento en Mendoza. Recuperado de: http://www.aaep.org. ar/espa/anales/works05/perlbach_calderon_riosrolla.pdf

PIÑA, L.I. (1977). La grana o cochinilla del nopal. Monografías LANFI NO. 1. Publicaciones de los Laboratorios Nacionales de Fomento Industrial México, pp. 55.

Portes, A. and SenSENBREnNer, J. (1993). Embeddedness and immigration: notes on the social determinants of economic action, The American Journal of Sociology, 98(6), 1320-1350.

Putnam, R. (2001). Social Capital: Measurement and Consequences. Canadian Joumal of Policy Research. 2, 41-51. Spring.

Rodríguez, P. \& Román del Río, C. (2005). El capital social como factor de competitividad y desarrollo empresarial. Revista Ekonomiaz, 59(20). Cuatrimestre, 214-231.

RUBen, R. y HeRAS, J. (2012). Social Capital, Governance y Performance of Ethiopian Coffee Cooperatives. Annals of Public and Cooperative Economics. 83:4. 
SUNKEL, G. (2001). La pobreza en la ciudad: capital social y políticas públicas. Documento preparado para la conferencia En busca de un nuevo paradigma: capital social y reducción de la pobreza en América Latina y el Caribe, CEPAL, Universidad del Estado de Michigan, Santiago de Chile, 24 al 26 de septiembre de 2001.

Tovar, P.A. (2000). Producción de grana cochinilla del nopal Dactylopius coccus Costa (Homoptera: Dactylopiidae) en dos localidades del sur del estado de Nuevo León. Tesis de Maestría como requisito parcial para obtener el grado de Maestro en Ciencias Forestales. Universidad Autónoma de Nuevo León. Recuperada de: http://eprints.uanl.mx/840/1/1020145908.PDF

VAlentinOV, V. (2003). Transition in agriculture, and economic organization: a theoretical perspective, Discussion Paper No. 53, Halle: Institute of Agricultural Development in Central and Eastern Europe (IAMO).

Woolcock, M. (1998). Social capital and economic development: toward a theoretical synthesis and policy framework, Theory and Society, 27, 151-208. 